\title{
Therapie und Prophylaxe von Dekubitalulzera - Teil 1
}

\author{
M. Schempf ${ }^{7}$, C. Warda 2 , M. Mentzel' ${ }^{3}$ Y.-B. Kalke ${ }^{4}$, K. Huch ${ }^{4}$ \\ 1 Nova Clinic Biberach an der Riß \\ 2 Donau-Ries Klinik Donauwörth \\ 3 Universitätsklinikum Ulm, Klinik für Unfall-, Hand-, Plastische und Wiederherstellungschirurgie \\ 4 Orthopädische Universitätsklinik Ulm am RKU
}

„Dekubitus“ wird allgemein als Kurzform des Wortes Dekubitalulkus (Druckgeschwür) gebraucht. Bei einem Dekubitalulkus handelt es sich um eine der folgenschwersten Komplikationen einer Immobilität. Für betroffene Patienten bedeutet dies eine schwere Erkrankung mit z. T. letalem Ausgang. Das Nichtauftreten eines Dekubitus wird als wichtiges Qualitätsmerkmal der Pflegeleistung angesehen und wurde seitens der Bundesgeschäftsstelle Qualitätssicherung (BQS) zum „Patientensicherheitsindikator“ erhoben.

Es wird geschätzt, dass pro Jahr mindestens 0,5\% der Bevölkerung betroffen sind. Bei Krankenhauspatienten findet man in bis zu $11 \%$ und bei Heimpatienten oder in häuslicher Pflege in 18-33\% der Fälle einen Dekubitus. Die volkswirtschaftliche Bedeutung ist aufgrund eines enormen Pflegeund Kostenaufwands groß.
Die Therapie erfolgt stadienabhängig: Das Frühstadium mit Rötung der Haut kann durch eine konservative Therapie behandelt werden. Kommt es allerdings zu einer weiteren Ausdehnung des Dekubitus mit tiefen Wundhöhlen und freiliegendem Knochen, wird eine aufwendige Therapie mit mehreren konditionierenden operativen Eingriffen mit anschließender großflächiger plastischer Deckung erforderlich. Diese wird im zweiten Teil des Beitrags behandelt

Die Therapie der Dekubitalulzera ist einerseits für die Betroffenen sehr belastend und andererseits zeitaufwendig und teuer. Somit ist nicht nur aus medizinischer und pflegerischer Sicht, sondern auch aus ethischer und ökonomischer Perspektive eine konsequente Prophylaxe essenziell.

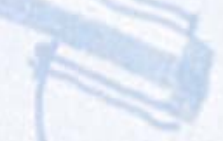

\section{Abkürzungen}

$\begin{array}{ll}\text { AZ } & \text { Allgemeinzustand } \\ \text { BQS } & \text { Bundesgeschäftsstelle Qualitätssicherung } \\ \text { EZ } & \text { Ernährungszustand } \\ \text { NICE } & \text { National Institute for Health and Clinical Excellence } \\ \text { TFL } & \text { Tensor-fasciae-latae-Lappen }\end{array}$

In einer Schweizer Studie konnte im Akutkrankenhaus eine Inzidenz eines Dekubitus 1. Grades oder höher in $10 \%$ der Patienten verzeichnet werden.

In Glasgow konnte bei 252 geriatrischen Patienten in $41 \%$ ein Dekubitus festgestellt werden. Man stellte fest, dass davon $45 \%$ vermeidbar gewesen wären. 


\section{Grundlagen}

Tabelle 1

Dekubitushäufigkeit in der internationalen Literatur (nach Gesundheitsberichterstattung des Bundes 2002, Heft 12).

\begin{tabular}{|c|c|c|}
\hline Quelle & Einrichtung & Prozent \\
\hline Allman 1986 & Krankenhaus & 4,7 \\
\hline EQS-Hamburg 1998 & Krankenhaus & 6,6 \\
\hline HPG Hamburg 1999 & ambulante Pflege & 8,9 \\
\hline Bergström 1992 & Krankenhaus & 9,0 \\
\hline HPG Hamburg 1999 & Pflegeheim & 11,2 \\
\hline Hamburg 1998 & Rechtsmedizin, Verstorbene & 11,2 \\
\hline Clark 1989 & Pflegeheim & 14,2 \\
\hline Berlin 2000 & Rechtsmedizin, Verstorbene & 16,2 \\
\hline Hunt 1993 & Intensivpatienten & 20,0 \\
\hline Hunter 1992 & Rehazentrum & 25,0 \\
\hline Bergström 1987 & Intensivpatienten & 40,0 \\
\hline
\end{tabular}

Gesundheitspolitisch ist der Dekubitus relevant, da er mit einem erhöhten Morbiditäts- und Mortalitätsrisiko assoziiert ist. Das Risiko, als Patient mit Dekubitus zu versterben, ist auf das 4-6-Fache erhöht. In den USA wurde zwischen 1990-2001 bei 114380 Patienten ein Dekubitus als Todesursache angegeben, dies entspricht einer Mortalitätsrate von 3,79 Personen/100000 Einwohnern.

Man schätzt, dass in Deutschland insgesamt pro Jahr mehr als 400000 Personen an einem Dekubitalulkus erkranken. Davon leiden ca. 150000 an einem Grad-IIIoder -IV-Dekubitus. Die Tendenz ist aufgrund der zunehmenden Zahl an alten und hoch betagten Menschen mit eingeschränkter Mobilität und oft ausgeprägter Multimorbidität ansteigend. Gerade bei diesem Patientenkollektiv stellt die Behandlung eines Dekubitus eine Herausforderung dar, die nicht selten unbewältigt bleibt.

Aus ökonomischer Sicht ist eine Prophylaxe unabdingbar, da hiermit 90\% der Kosten für das Gesundheitswesen gespart werden könnten. Die Berechnung der Kosten ist schwierig, da viele Faktoren mit eingerechnet werden müssen, wie z.B. Materialkosten, Personalkosten, Kosten für verlängerten Aufenthalt und Folgeschäden usw. Es werden in der Literatur unterschiedliche Liegedauern von 27 Tagen bis 6 Monate angegeben. Dabei entstehen Kosten von 15000 - 25000 Euro pro Patient. 1998 wurden in Deutschland 16 Mio. Patienten stationär behandelt. Bei einer Inzidenzrate von 1,3\% entwickelten somit 207000 Patienten einen Dekubitus. Daraus resultieren 1,1 Mio. zusätzliche Pflegetage mit Mehrkosten von 200 Mio. Euro, was ca. 10000 Euro pro Patient entspricht.

\section{Ätiologie}

\section{Definition}

\section{Dekubitus}

Ein Dekubitus ist definiert als Schädigung der Haut und des Unterhautgewebes durch eine anhaltende lokale Druckeinwirkung.

Bei seiner Entstehung übt der menschliche Körper beim Sitzen oder Liegen Druck auf die Auflagefläche aus, die ihrerseits auf das aufliegende Hautareal einen Gegendruck erzeugt.

Abhängig von der Härte der Auflagefläche wird der Gegendruck normalerweise über dem physiologischen Kapillardruck von ca. 25-35 mmHg arteriell liegen.

Kurzfristig kann die Haut selbst höhere Druckeinwirkungen tolerieren.

Wird dieser Druck jedoch länger aufrechterhalten, werden Kapillaren komprimiert, und es kommt durch eine Minderperfusion des Gewebes zur Hypoxie. Durch die Minderdurchblutung kommt es zu einer Anhäufung toxischer Stoffwechselprodukte mit Erhöhung der Kapillarpermeabilität, Gefäßerweiterung, zellulärer Infiltration und Ödem und somit zur kompletten Ischämie und zum Absterben der Hautzellen.

Auf eine beginnende Schädigung der Haut reagiert der gesunde Körper mit einem Druckschmerz, der zu einer Lageänderung mit Entlastung des betroffenen Hautareals (auch im Schlaf) führt, wodurch es zu einer Reperfusion der Gewebeschichten kommt (Abb. 1).

Ist ein Mensch nicht in der Lage, den Druckschmerz wahrzunehmen, z.B. bei Bewusstlosigkeit, Querschnittsyndrom, Narkose, schweren Demenzen und/oder ist er nicht mehr fähig, sich aus eigener Kraft aufgrund des Druckschmerzes zu bewegen, so bleibt die Komprimierung des Hautareals bestehen. 

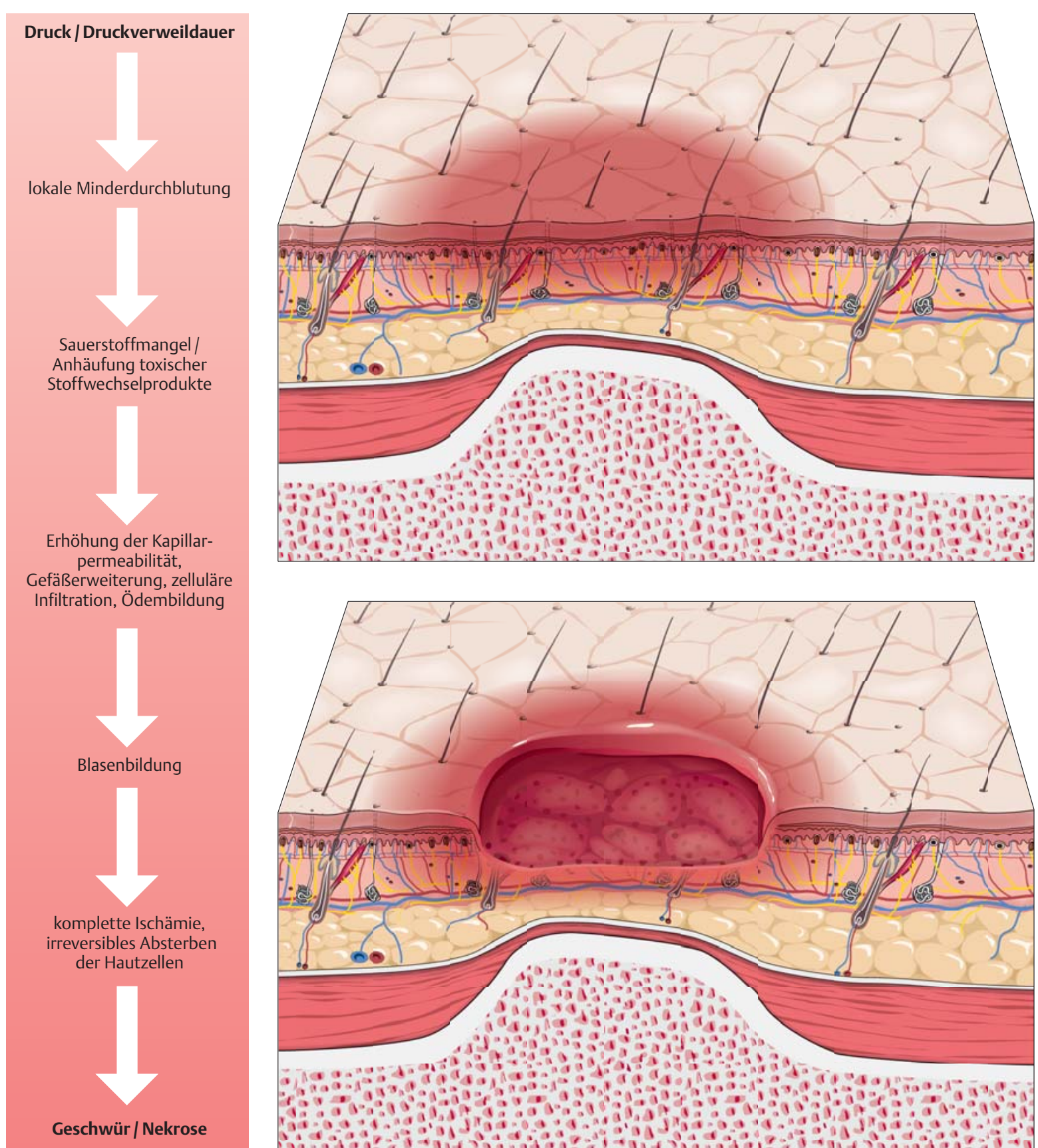

Abb. 1 - Entstehung eines Dekubitus.

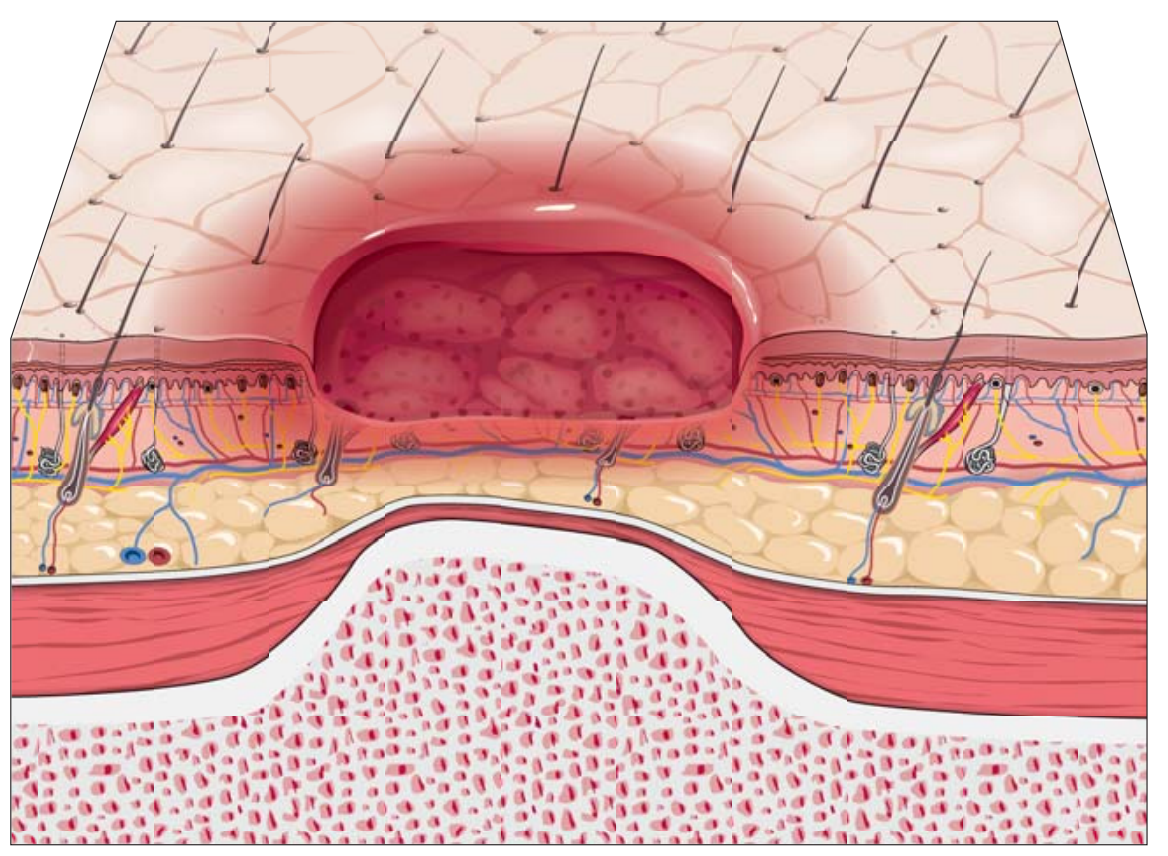

- Die Faktoren Druck und Zeit sind für die Entstehung eines Dekubitus maßgeblich verantwortlich.

Ein hoher Druck führt früher zum Gewebeschaden als ein niedriger. Allerdings können hohe Druckwerte von kurzer Dauer gut von der Haut toleriert werden. Wird dennoch ein (geringfügiger) Druck über längere Zeit aufrechterhalten, kann nach wenigen Stunden bereits ein Dekubitalulkus entstehen.
Nicht nur die senkrechte Druckeinwirkung ist Ursache eines Druckulkus, auch Scherkräfte in der Haut können Ulzerationen verursachen. Prädilektionsstelle ist hier die Glutäalregion, da beim Transfer eines Patienten dieser häufig nicht angehoben, sondern gezogen wird. So zeigte sich bei experimentellen Untersuchungen, dass bei der Kombination aus Druck und tangentialen Scherkräften bereits ein niedrigerer Druck genügt, um den Sauerstoffpartialdruck im Gewebe auf einen kritischen Wert abzusenken (Bennett et al. 1984, von Goossens et al. 1994). 


\section{Grundlagen}

Der durchschnittliche Toleranzbereich der Haut gegenüber Druckbelastung und der Zeit der Druckeinwirkung (30-240 min) ist interindividuell sehr verschieden und wird durch bestimmte Risikofaktoren begünstigt, was das Geschehen sehr komplex und schwer fassbar macht (Tabelle TA1).

\section{Risikofaktoren}

Eine Risikogruppe stellen die Patienten mit einer krankheitsbedingten Immobilität dar. Dies sind insbesondere ältere, multimorbide Patienten, die mit 60\% das wichtigste Kollektiv der Dekubituserkrankten darstellen. Durch altersbedingte Erkrankungen haben ältere Patienten weniger Reserven als vergleichsweise jüngere Patienten mit gleicher Erkrankung, des Weiteren ist der willkürliche Lagewechsel im Alter herabgesetzt. Weitere Hochrisikopatienten sind Querschnittgelähmte, die aufgrund ihrer Grunderkrankung zum Dekubitus neigen. Auch Frühgeborene können durch noch nicht ausgebildete Eigenbeweglichkeit einen Dekubitus entwickeln.

Nach der NICE-Empfehlung (National Institute for Health and Clinical Excellence) gibt es ein Minimum an Risikofaktoren, die beachtet werden müssen (siehe Checkliste).

Des Weiteren werden permanente, häufige und mögliche Faktoren für die Entstehung von Dekubitalulzera angegeben (Tab. 2).

\section{Tabelle 2}

Faktoren zur Entstehung eine Dekubitus.

\begin{tabular}{|c|c|c|}
\hline Permanent & Häufig & Möglich \\
\hline Immobilität & Hyperthermie & Ernährungszustand \\
\hline Inkontinenz & vegetative Transpiration & Stoffwechselerkrankungen \\
\hline \multirow[t]{6}{*}{ Sensibilitätsveränderung } & Spastik & Dehydratation \\
\hline & Reibung/Scherkräfte & schlechter Allgemeinzustand \\
\hline & Depression & fehlende Compliance \\
\hline & & Durchblutungsstörung \\
\hline & & Kontrakturen \\
\hline & & Skelettdeformitäten \\
\hline
\end{tabular}

\section{Checkliste}

\begin{tabular}{|l|}
\hline Risikofaktoren nach der NICE-Empfehlung \\
\hline Alter \\
\hline Mobilität \\
\hline Aktivität \\
\hline Bewusstseinsgrad \\
\hline Ernährung \\
\hline Kontinenz \\
\hline Hautzustand \\
\hline Krankheitsschwere \\
\hline
\end{tabular}

\section{Primäre Risikofaktoren}

Bei totaler Immobilität ist der Patient absolut gefährdet (z.B. bei Bewusstlosigkeit, Narkose, vollständige Lähmung). Das Alter des Patienten spielt dabei keine wesentliche Rolle.

Bei relativer Immobilität besteht ein hohes Gefährdungspotenzial, weil Spontanbewegungen mehr oder weniger eingeschränkt sind, beispielsweise durch Sedierung, bei Frakturen, starken Schmerzzuständen, Multipler Sklerose, Querschnittlähmung, Halbseitenlähmung und Sensibilitätsstörungen unterschiedlichster Ursachen, wie z.B. einer Polyneuropathie.

Es entstehen typischerweise nachts kritisch lange Immobilitätsphasen, da am Tage durch Grundpflege und Nahrungsaufnahme eine gewisse Mobilisation stattfindet. Dies wird insbesondere bei alten Menschen mit reduzierten Spontanbewegungen oder bei Immobilisation durch Fieber oder Schmerzen verstärkt.

\section{Sekundäre Risikofaktoren}

Hierunter fallen alle Krankheitsbilder, welche die Funktionsfähigkeit und Widerstandskraft der Haut herabsetzen; somit genügen kurzzeitige Druckeinwirkungen, um eine Ulzeration zu verursachen:

- Mangeldurchblutung der Haut: Dies bedeutet eine reduzierte Sauerstoffversorgung des Gewebes und einen damit einhergehenden veränderten Zellstoffwechsel (z.B. durch hypovolämischen, kardiogenen oder septischen Schock, niederen Blutdruck, Dehydratation, Herzinsuffizienz, Diabetes mellitus, Arteriosklerose usw.). 
- Fieber: Dabei kommt es zum gesteigerten Stoffwechsel und erhöhten Sauerstoffbedarf der Hautzellen.

- Inkontinenz: Durch Feuchtigkeit und aggressive Zersetzungsprodukte von Urin und/oder Stuhl wird die Haut aufgeweicht und kann zusätzlich stark bakteriell belastet sein. Wird keine suffiziente Hautpflege durchgeführt, kommt es zu Mazeration und herabgesetzter Widerstandskraft der Haut. Allerdings ist es falsch, dass Urin alleine einen Dekubitus verursachen kann, da Druck immer Voraussetzung für die Entstehung ist. Urin wird als Kofaktor gewertet.

- Reduzierter Allgemeinzustand: Durch chronisch verlaufende oder schwere Krankheiten, maligne Prozesse, Infektionen, Malnutrition mit Eiweiß-, Vitamin- und Zinkmangel, Anämie, Exsikkose, Kachexie wird die Mobilität der Patienten herabgesetzt.

- Physiologische Hautalterung: Die Altershaut verliert mechanische Belastbarkeit, durch Verlust von Zellund Faserelementen. Die Haut wird dadurch dünner und verliert an Elastizität.

\section{Operationsspezifische Risiken}

Auch für einen intraoperativ erworbenen Dekubitus gilt das Grundprinzip: Druck, der über eine gewisse Zeit wirkt. Die Risiken sind operationsabhängig und patientenspezifisch. Operationsspezifische Risiken können sein:

- narkosebedingter Tonusverlust der Haut,

- fehlerhafte Umlagerungen, vor allem der Extremitäten,

- extreme Scherkräfte und Druckbelastung bei der Frakturbehandlung auf dem Extensionstisch,

\section{Risikofaktoren}

\section{Primäre Risikofaktoren, welche die Motilität} vermindern und zur totalen/relativen Immobilität führen (nach Seiler 2002)

Neurologische Krankheiten mit Lähmungen (alle):

- zerebrovaskulärer Insult, Hemiplegie, Hemiparese, Paraplegie, Tetraplegie, komatöse Zustände jeder Genese

\section{Chirurgische Eingriffe:}

- Anästhesie (Prämedikation, Narkose, Aufwachphase), lange Operationszeiten

Psychiatrische Krankheiten und Psychopharmaka:

- akute Psychosen wie Katatonie und akute Depressionen, sedierende Medikamente wie Neuroleptika, Benzodiazepine und ähnliche konsumierende Erkrankungen und starke Schmerzzustände
- sich aufstützende Assistenten,

- Unterkühlung des Patienten,

- fehlerhafte Anwendung von Desinfektionsmitteln,

- lange Gefäßklemmzeiten,

- Blutleeren.
Sekundäre Risikofaktoren, die insbesondere die Gewebetoleranz herabsetzen (nach Seiler 2002)

Faktoren, die den intravaskulären Druck vermindern:

- arterielle Hypotension: Schock (hypovolämisch, septisch, kardiogen), Antihypertensivaüberdosierung

- Dehydration: Diuretika, Diarrhö, Sommerhitze

Faktoren, die den Sauerstofftransport zur Zelle vermindern:

- Anämie: Hämoglobin < $9 \mathrm{~g} / \mathrm{dl}$

- periphere arterielle Verschlusskrankheit

- diabetische Mikroangiopathie

- Hypotonie, Bradykardie

Faktoren, die den Sauerstoffverbrauch in der Zelle erhöhen:

- hypovolämischer Schock

- Fieber: $>38^{\circ} \mathrm{C}$

- Hypermetabolismus

- Infektionen, Zytokinämie

Faktoren, die zu Nährstoffmangel in der Zelle führen:

- Malnutrition: Mangel an Eiweiß, Vitaminen, Mineralstoffen, Spurenelementen

- Kachexie: Immobilität durch Muskelschwäche und Katabolismus

- Lymphopenie bei Malnutrition: Immunschwäche, Störung der Wundheilung

Faktoren, die den Widerstand der Haut schwächen

- Altershaut: dünn, atrophisch, mit weniger Abwehrzellen

- Hautkrankheiten: Ekzeme, Soorbefall

- trockene, rissige Haut: begünstigt Hautinfektionen mit Bakterien und Pilzen

- druckgeschädigte, gerötete Haut: als Zeichen der schädlichen Shuntzirkulation

- mazerierte, aufgeweichte Haut: bei Inkontinenz durch Zersetzungsprodukte von Urin und Stuhl

- Hitze, entzündliche Rötung: Umgehung der nutritiven Mikrozirkulation

- steroidinduzierte Hautatrophie: dünne, leicht verletzliche Haut 


\section{Diagnostik}

\section{Symptomatik}

Die primäre Symptomatik einer Druckbelastung ist der Schmerz. Aufgrund einer Grundkrankheit kann ein Lagewechsel ggf. nicht selbstständig vom Patienten durchgeführt werden (z. B. bei inkompletten Querschnittlähmungen mit erhaltener Sensibilität). Bei bestimmten Krankheiten werden auch keine Schmerzen verspürt, sodass kein Stimulus zum Lagewechsel vorhanden ist. Die anfängliche Rötung der Haut oder das nachfolgende manifeste Ulkus sind Leitsymptome.

\section{Diagnostik und Dokumentation}

- Anamnese

- Alter des Patienten,

- Entstehungsursache mit „dekubitusfördernden Faktoren und Krankheitsbildern“,

- Nebenerkrankungen,

- Medikamentenanamnese,

- Voroperationen,

- Vordiagnostik.

\section{- Risikoprofil erstellen}

- Körperlicher Allgemeinzustand: Es sollte hierbei insbesondere auf mögliche Komplikationen, die mit einem Dekubitus in Verbindung stehen, geachtet werden (z.B. Endokarditis, Meningitis, septische Arthritis, Taschen- und Abszessbildung, maligne Entwicklung des Ulkus, Jod-Toxizität).

- Ernährungsstatus: Es besteht eine schlechte Heilungstendenz eines Dekubitus bei Malnutrition mit Kachexie und Einweißmangel. Hier ist auf eine ausreichende Nahrungs- und Flüssigkeitszufuhr mit Eiweißen, Vitaminen und Mineralien zu achten. Dies ist ggf. mit i.v. Nahrung oder Sondenkost mit PEG-Anlage zu gewährleisten.

- Schmerzen: Selbst wenn der Patient seine Schmerzen nicht äußern oder darauf reagieren kann, können diese vorhanden sein und sich als diffus erweisen. Vor allem Lagewechsel sind für den Patienten stark schmerzbelastend. Es reicht nicht nur, eine Bedarfsmedikation anzusetzen, sondern es sollte eine individuelle, kontinuierliche Schmerztherapie durchgeführt werden.

- Psychosoziale Beurteilung: Voraussetzung für die Dekubitustherapie ist die Mitarbeit des Patienten, die von der mentalen Fähigkeit oder Lernfähigkeit abhängt.

- Beurteilung der Dekubitalulkusgefährdung: Zur Planung der Prophylaxe eines Dekubitus ist die individuelle Einschätzung eines jeden Patienten Voraussetzung.

Zur Beurteilung der Risikofaktoren stehen verschiedene Beurteilungsskalen zur Verfügung. Diese Risikoskalen dienen zur Identifikation von Patienten, die für Druckgeschwüre besonders anfällig sind. Die Leitlinien des britischen NICE geben vor, dass neben den Skalen das ganzheitliche Individuum eingeschätzt werden solle.

In Deutschland werden hierzu überwiegend der NortonSkala (Tab. TA1, siehe Anhang) und Braden-Skala (Tab. TA2, siehe Anhang) zur Klassifizierung herangezogen.

Die Skalen berücksichtigen den geistigen und körperlichen Zustand, sowie die Aktivität und Beweglichkeit des Patienten. Wichtig ist die regelmäßige Wiederholung der Beurteilung, um rechtzeitig Veränderungen festzustellen und zu dokumentieren.

\section{- Inspektion und Wundbeurteilung}

Bei der Wundinspektion sollten der Schweregrad des Dekubitus festgelegt werden sowie Lokalisation, Größe/ Tiefe/Taschenbildung/Fistel, Exsudation, Wundstadium, Geruch, Wundrand/-umgebung, Infektionszeichen, Hautzustand und das Alter der Wunde beurteilt werden.

\section{Qualitäten der Wundbeläge}

- Schwarz: Nekrose,

- rot: Granulation,

- gelb: Eiter, Detritus, Fibrinbeläge,

- übler Geruch bei einer Rötung der Wunde und Überwärmung sprechen für eine akute Infektion.

Dekubituslokalisation. Prinzipiell kann an jedem Hautareal ein Dekubitus entstehen, abhängig von der Lagerung eines Patienten. Prädilektionsstellen sind allerdings Bereiche, bei denen der Druck senkrecht auf das Hautareal einwirkt. Dies sind insbesondere Stellen der konvexen Skelettbereiche mit wenig druckverteilendem, elastischem Muskel- und Unterhautfettgewebe. Es ist zu erwähnen, dass der Druck über konvexen Knochenkonturen von der großen Hautoberfläche in die Tiefe auf die kleinere konvexe Knochenfläche hin zunimmt. Dabei entstehen primär die Nekrosen im druckempfindlicheren Unterhautfettgewebe und in der Muskulatur, bis sich die Ulzerationen nach einer Latenzzeit von Tagen 


\section{Therapie und Prophylaxe von Dekubitalulzera - Teil 1}

\section{Hintergrundbox}

Abb. 2 Druckschädigung in Abhängigkeit von der Position des Patienten.

1. In Bauchlage: Stirnknochen, Ellbogen, Brustbein- und Rippenbögen, Beckenkamm, Kniescheiben und Zehenspitzen

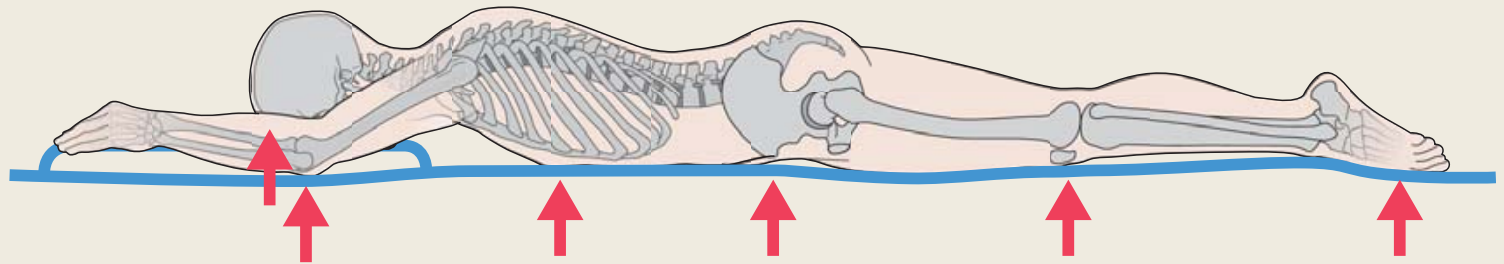

2. In Rückenlage: Kreuzbein und Steißbein, Fersen und Achillessehnen, Ellbogen, Schulterblätter und Hinterhauptknochen

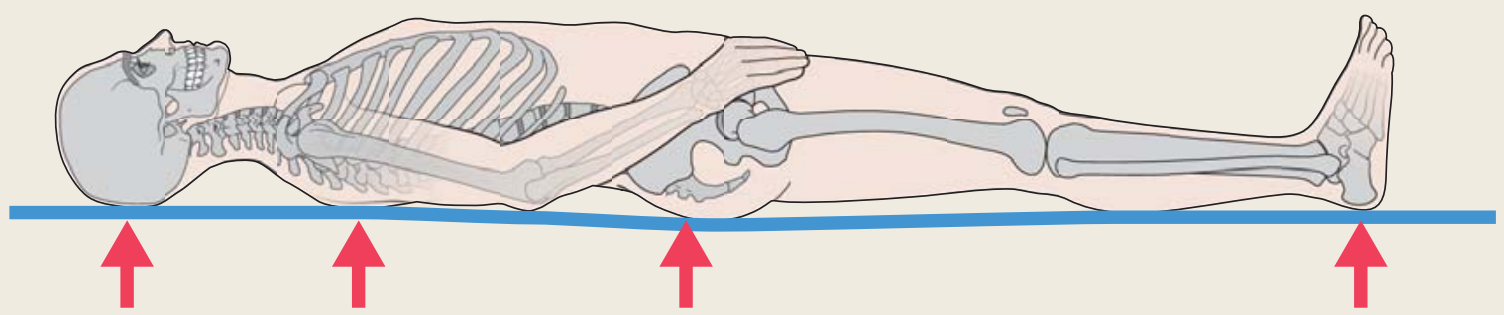

3. In Seitenlage: großer Rollhügel (Trochanter major), Ohren- und Jochbeinknochen, seitliche Rippenanteile, Schultergelenk, Beckenkamm, inneres und äußeres Kniegelenk, Wadenbein und seitlicher Knöchel

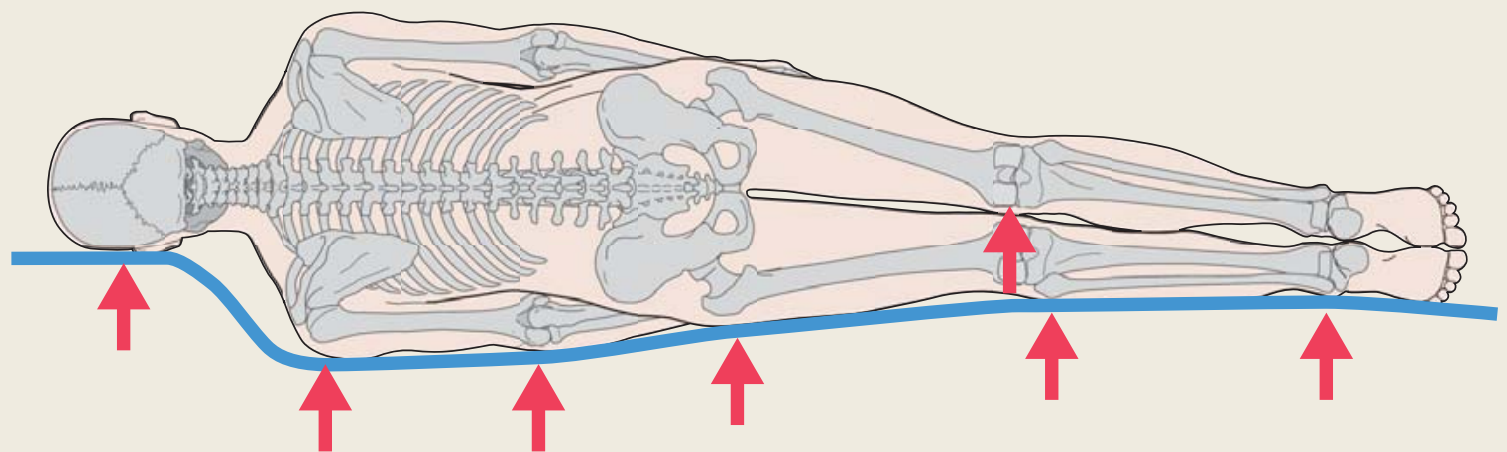

4. In sitzender Position: Sitzbeinhöcker, Hinterhauptknochen, Wirbelsäule und Fersen; bei schlechter Abstützung ergibt sich hier zusätzlich eine Gefährdung durch Scherkräfte

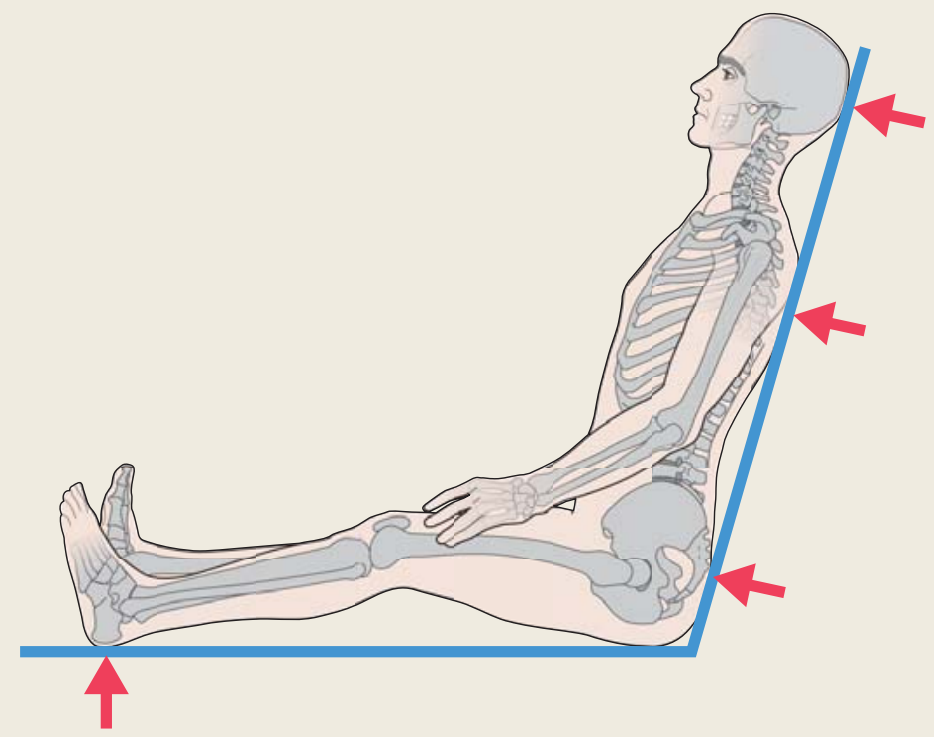


(3-10 Tage) durch Hautnekrosen bemerkbar machen. Somit ist von der Hautseite nicht immer das gesamte Ausmaß der Destruktion zu erfassen, da die Schädigung des Unterhautgewebes in der Tiefe weitaus größer sein kann - „Die Haut ist nur die Spitze des Eisberges.“

95\% aller Dekubitalulzera entstehen im Sakralbereich, an den Fersen, Sitzbeinen, Trochanter major und laterale Malleolen (Abb. 2).

Dekubitusklassifizierung. Der Dekubitus wird in der Regel in 4 Grade oder Stadien eingeteilt, wobei die Klassifikation der Schweregrade nach der Tiefenausdehnung auf Shea et al. 1975 zurückzuführen ist. Abb. 3 verdeutlicht die verschiedenen Grade der Dekubitalulzera bezüglich der Tiefen und der 3-dimensionalen Ausdehnung. Es existieren unterschiedliche Einteilungen, wie in Tab. TA3, TA4, TA5 und Abb. 3 aufgeführt, wobei sich in Deutschland die Klassifikation nach Daniel und Seiler etabliert hat.

Differenzialdiagnostisch sind andere Hautveränderungen, wie wegdrückbare Erytheme oder chronische Wunden anderer Genese (diabetisches Ulkus, Ulcus cruris venös bedingt) auszuschließen.
Höhergradige Druckgeschwüre über den Sitzbeinen oder den Trochanteren können in das Hüftgelenk einbrechen und enden nicht selten über ein Gelenkempyem in einer Hüftkopfresektion (Girdlestone-Situation). Auch die Penetration ins Rektum oder die Harnröhre bzw. Symphyse wurde beschrieben.

Zur Bestimmung der Ausdehnung des Ulkus wird das „Litern“ angewandt. Hierbei wird der Ulkus mit einer Folie abgeklebt und mit steriler Flüssigkeit ausgegossen, womit man die Volumenausdehnung des Dekubitus bestimmt. Des Weiteren kann mit einer Messsonde die Ausdehnung erfasst werden.

\section{Präoperative Diagnostik}

- Labor: Hb, Gesamteiweiß, Albumin, $\mathrm{HbA}_{1 \mathrm{c}}, \mathrm{CRP}$, Leukozyten, Gerinnung.

- Röntgen (Beckenübersicht), ggf. Sonografie, CT, MRT: Hiermit können eine Osteitis oder Osteomyelitis, Skelettdeformitäten oder periartikuläre Verkalkungen verifiziert werden.

- Bei Fistelgängen ist ggf. eine Endoskopie in Form einer Rektoskopie indiziert.

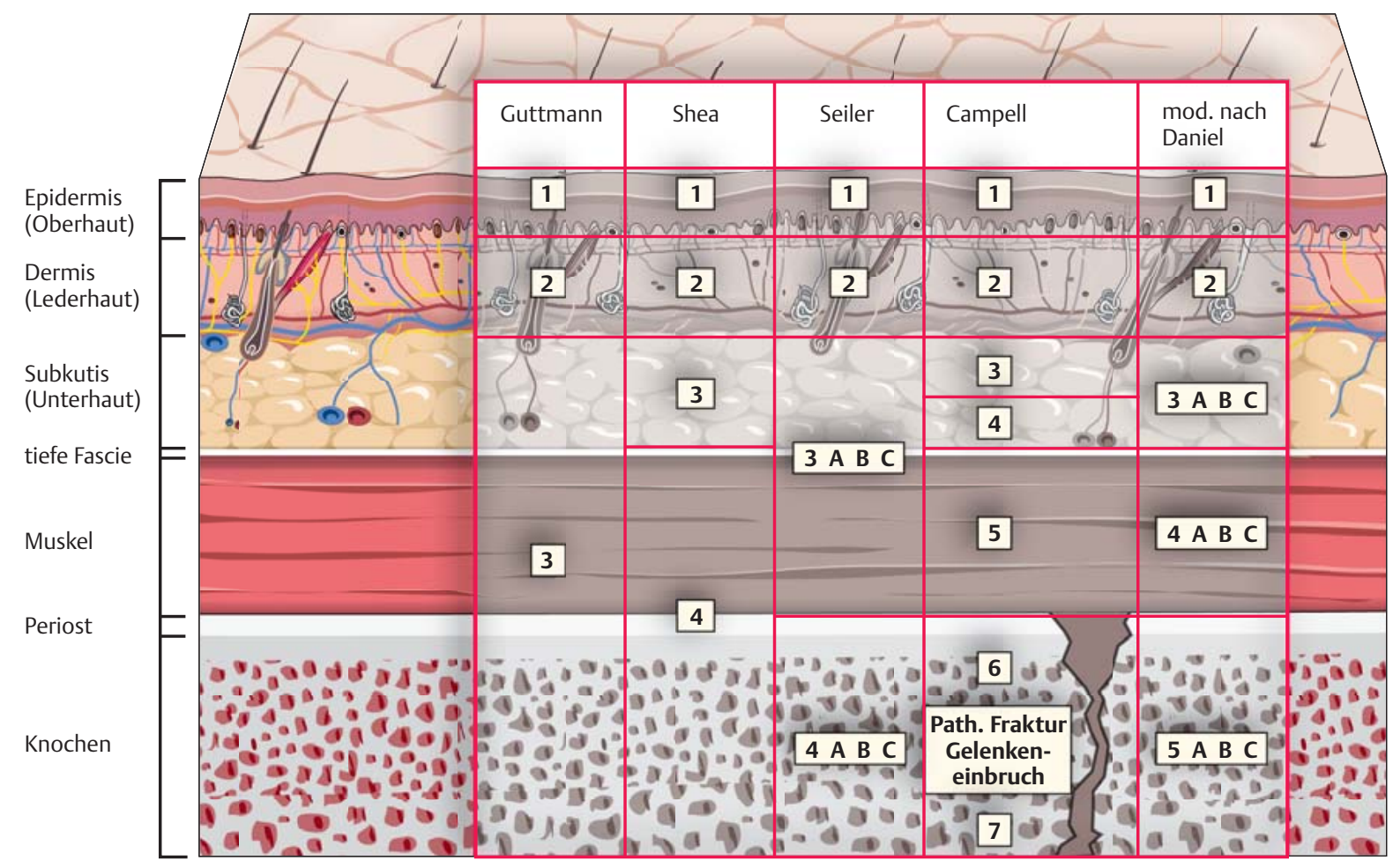

Abb. 3 - Vergleich der unterschiedlichen Stadieneinteilungen der Dekubitusausdehnung. 


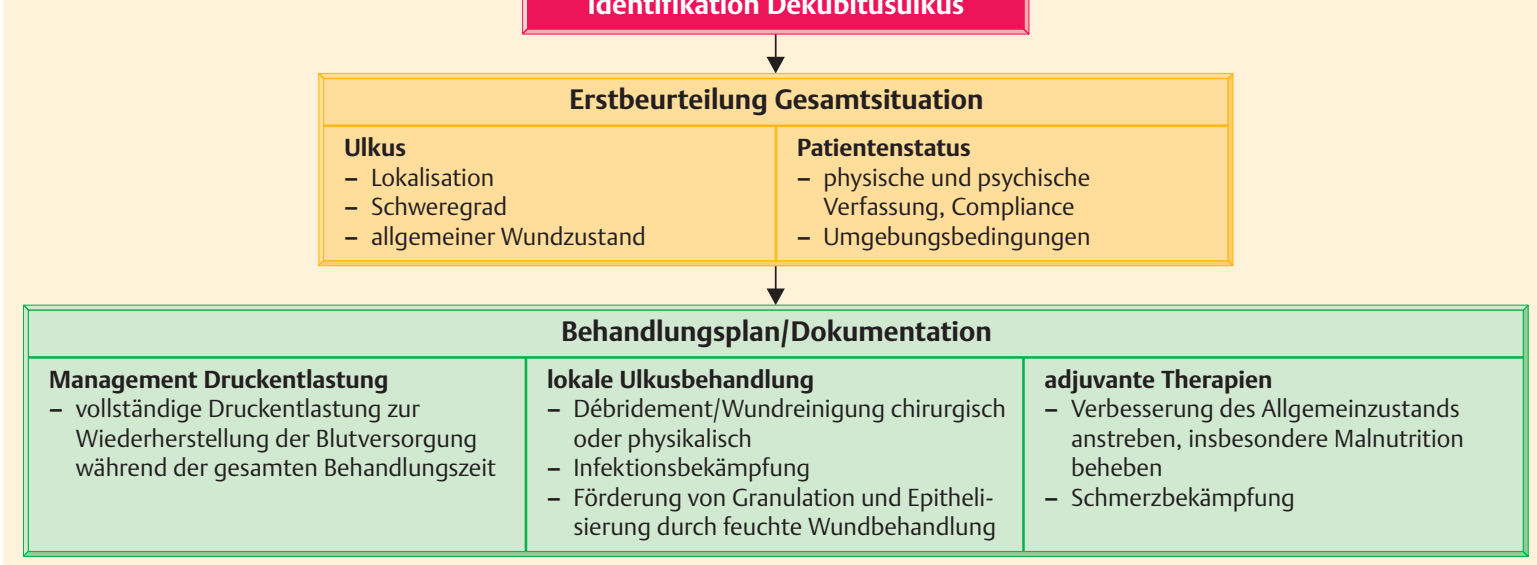

- Wundabstrich/Probebiopsie: Keimidentifizierung, MRSA-Ausschluss mit ggf. Beginn einer Antibiotikatherapie.

- Blutkulturen bei Fieberanstieg und Sepsis.

- Eine Angiografie zur Planung von gefäßgestielten Muskellappenplastiken ist empfehlenswert.

Es wird eine regelmäßige Schrift- und Fotodokumentation gefordert, um den Verlauf der Erkrankung zu kontrollieren oder zu verbessern.

\section{Therapie}

\section{Therapieziele}

Ziel sowohl der konservativen als auch der operativen Therapie eines Dekubitus ist die Wiederherstellung intakter, druckstabiler Haut- und Weichteilverhältnisse, die eine vollständige Mobilisation und Reintegration in den Alltag ermöglichen und nicht zuletzt die Lebensqualität des Patienten steigern.

Das wichtigste Therapieziel ist die Prophylaxe des Dekubitus. Dies bedeutet:

- Verkürzen der Druckverweilzeit durch regelmäßige, 2-stündige Lagerung in die Rechts/Links-30-Schrägseitenlage.

- Verminderung der Größe des Auflagedrucks an allen 5 klassischen Dekubituslokalisationen unterhalb $25 \mathrm{mmHg}$ durch weiche Matratzen.

- Verbesserung des Allgemeinzustands des Patienten durch Behandlung der primären und sekundären Risikofaktoren wie Malnutrition, entgleiste Blutdrucke,

\section{Prinzipien}

\section{Operative und konservative Prinzipien}

Je nach Schweregrad des vorliegenden Dekubitus wird die Indikation zu einem konservativen oder chirurgischen Vorgehen gestellt. Die erfolgreiche Behandlung eines Dekubitus basiert auf einem interdisziplinären Therapiekonzept, das 6 Behandlungsprinzipien enthält:

- Druckentlastung,

- Débridement,

- Wundkonditionierung,

- Risikofaktorenminimierung,

- plastische Chirurgie,

- Prophylaxe.

Hämoglobin, Albumin, Mineralstoffe, Vitamine. Vermeiden von Hypovolämie, Dehydratation. Fiebersenkung, Einstellung des Diabetes mellitus, Behandlung von Pneumonie, Depression. Therapie von Infektionen jeglicher Art, Einfetten trockener Hautareale. Reduktion sedierender Medikamente auf ein Minimum.

Die Kombination dieser 3 Methoden sollte routinemäßig angewandt werden.

Ist aber ein Dekubitus entstanden, muss alles getan werden, um eine Vergrößerung und Ausdehnung zu vermeiden, alle konservativen Maßnahmen auszuschöpfen und ggf. eine operative Sanierung durchzuführen, um eine voll belastbare Hautsituation zu erreichen. Rezidive sollten durch Minimierung der Risikofaktoren verhindert werden (Abb. 4).

Tab. 3 zeigt einen Behandlungsplan für konservative und operative Therapie. 


\section{Grundlagen}

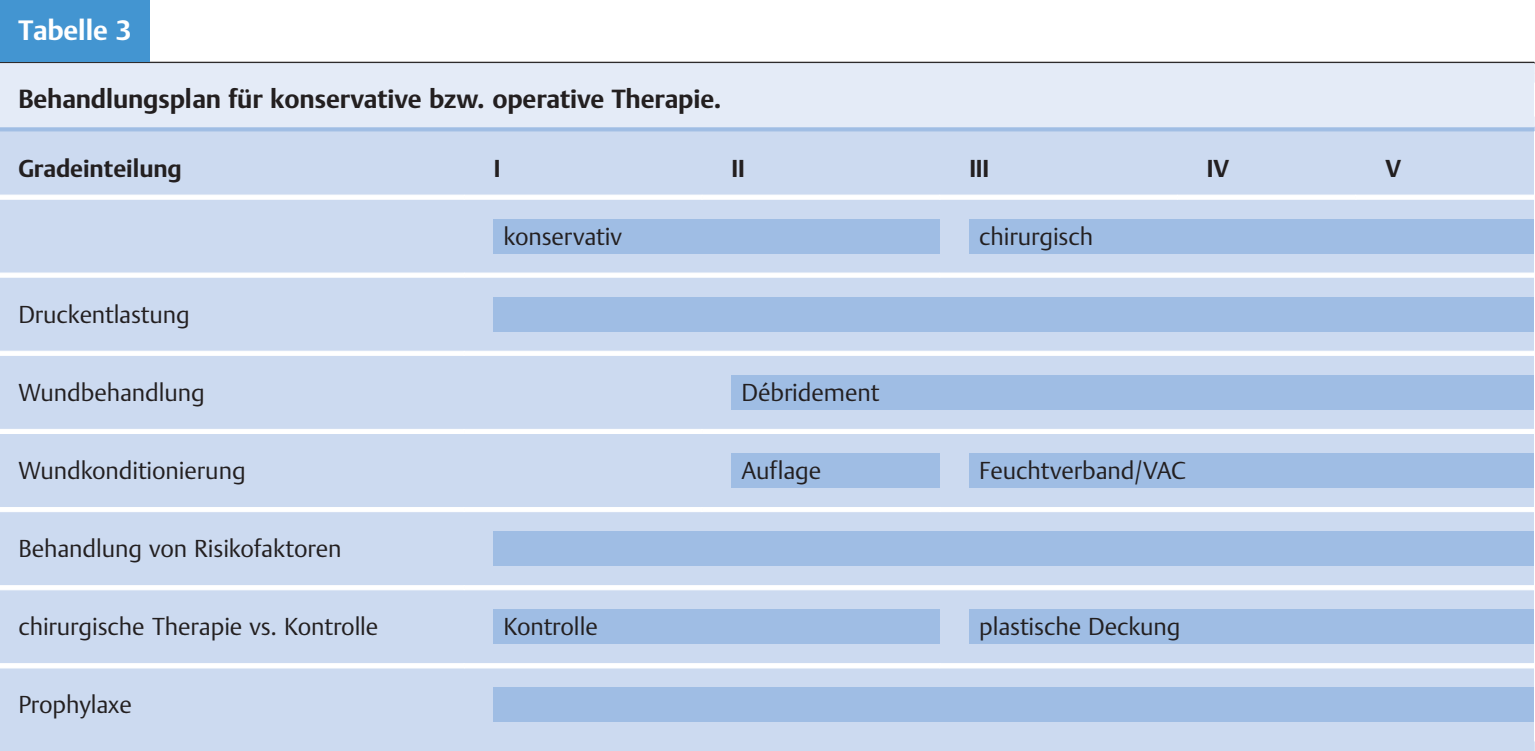

Prinzipien

\section{Stadiengerechte Dekubitusbehandlung}

Stadium I: Sofortige konsequente Druckentlastung nach individuellen Lagerungsplan. Wundbehandlung ist nicht erforderlich.

Stadium II: Auch hier sofortige Druckentlastung nach Lagerungsplan. Débridement und Reinigung der Wunde. Beachten von Infektzeichen. Ab Grad II benötigen alle Dekubiti Wundauflagen. Die Entfernung von Nekrosen ist Grundvorausetzung jeder Wundheilung, um damit v.a. eine Wundinfektion zu verhindern. So kann man ein Dekubitusstadium II und III über ein Stadium I sanieren. Bei Grad II kann die Wundreinigung durch enzymatisches oder autolytisches Débridement durch Hydrogele oder isotone salinische Lösungen durchgeführt werden. Dies ist insbesondere bei Hochrisiko- und multimorbiden Patienten von Vorteil.

Stadium III: Chirurgisches Abtragen von Nekrosen, denn unter Nekrosenkappen können sich eitrige Infekte verbergen, die in tiefere Schichten vordringen und ggf. zu Osteomyelitis führen können. Hierbei muss ein radikales Débridement und evtl. Knochenbiopsien bei V.a. Osteomyelitis durchgeführt werden.

Stadium IV: Die Behandlung erfolgt in der Regel chirurgisch mit Wundkonditionierung und anschließender Lappenplastik zur Wiederherstellung der Hautkontinuität.

\section{Konservative Therapie}

Die wesentlichen Säulen der konservativen Therapie sind:

- die Wundkonditionierung mit Wundsäuberungsphase,

- die Druckentlastung,

- das Überführen des Patienten in eine anabole Stoffwechsellage.

Wesentlich für die konservative Therapie eines Dekubitus sind die Beachtung von Risikofaktoren und die Behandlung dieser.

Bei der regelmäßigen Inspektion der Haut von Risikopatienten sollte besondere Aufmerksamkeit auf die Hautpartien im Bereich von Knochenprominenzen gelegt werden. Hier setzt die Kausaltherapie des Ulkus in Form einer konsequenten Druckentlastung ein. Die Druckentlastung ermöglicht eine Reperfusion des betroffenen Hautareals und führt somit zu einer verbesserten Sauerstoffsättigung des Gewebes. Die physiologische Druckverweilzeit liegt unter 2 Stunden. Die willkürlichen und unwillkürlichen Bewegungen bilden einen natürlichen Schutz vor einem Dekubitus. Bei jungen Menschen beträgt die Druckverweilzeit 15 Minuten, d.h. dass ein Patient 4-mal stündlich mobilisiert werden müsste. Die Erfahrung zeigte allerdings, dass durch ein 1- bis 2-stündliches Umlagern, auch von stark gefährdeten Patienten, eine suffiziente Dekubitusprophylaxe in 95\% gewährleistet werden kann. 


\section{Prinzipien}

Abb. 5 Verschiedene Lagerungstechniken.

Die bekannteste Lagerung ist die $\mathbf{3} 0^{\circ}$-Schräglage. Hierbei wird eine Belastung einer Gesäß- bzw. Körperhälfte herbeigeführt, da sich diese Stellen aufgrund von Polsterung durch Gewebe und fehlende Knochenvorsprünge zur Druckbelastung eignen. Von Vorteil z. B. bei der Lagerung von Schmerzpatienten ist es, dass der Patient kaum bewegt werden muss, um eine große entlastende Wirkung zu erreichen. Angewandt wird diese Lagerung bei Dekubiti von rechter oder linker Trochanter, Ferse, Os sacrum, Os ischii.

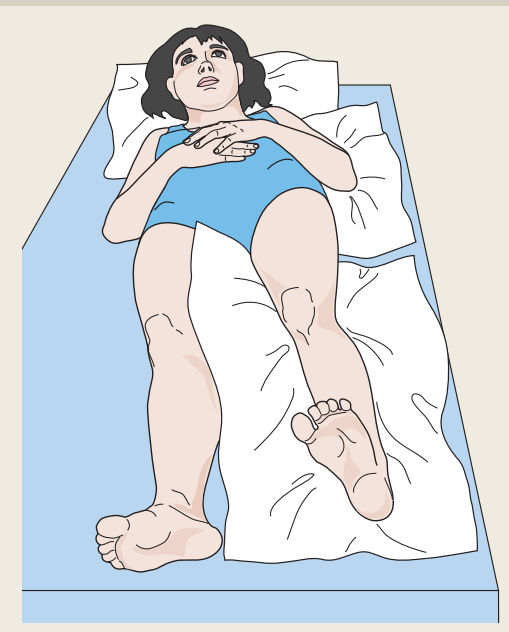

Bei der Freilagerung können betroffene oder gefährdete Körperstellen vollständig entlastet werden, z. B. Fersenulkus. Zu beachten ist eine mögliche Mehrbelastung einer angrenzenden Körperstelle.

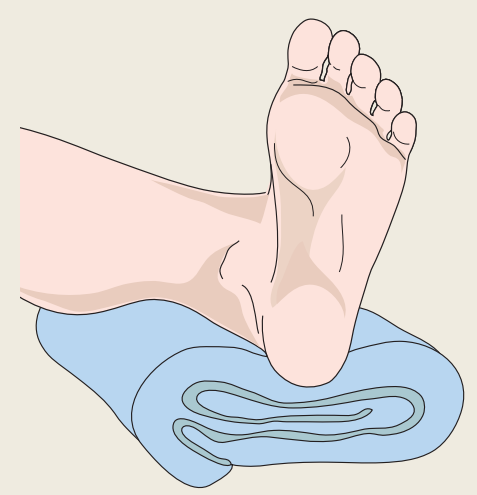

Eine weitere Lagerungstechnik ist die sog. $135^{\circ}$-Lagerung. Sie wird bei bereits bestehenden Druckgeschwüren am Os sacrum, Os ischii verwendet, da diese durch diese Lagerung größtenteils entlastet wird. Bei der Freilagerung können betroffene oder gefährdete Körperstellen vollständig entlastet werden, z.B. Fersenulkus. Zu beachten ist eine mögliche Mehrbelastung einer angrenzenden Körperstelle.
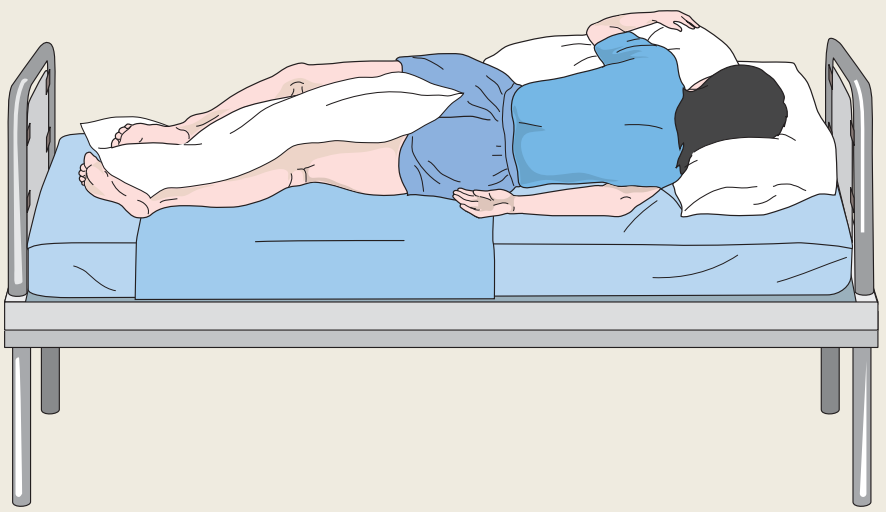

Eine spezielle Form der Lagerung stellt die Mikrolagerung dar. Sie setzt eine gewisse Mobilität des Patienten voraus. Bei dieser Lagerung wird durch kleinste Schwerpunktverlagerungen eine prophylaktische Wirkung erzielt. Sie ist als therapeutische Lagerung nicht geeignet.

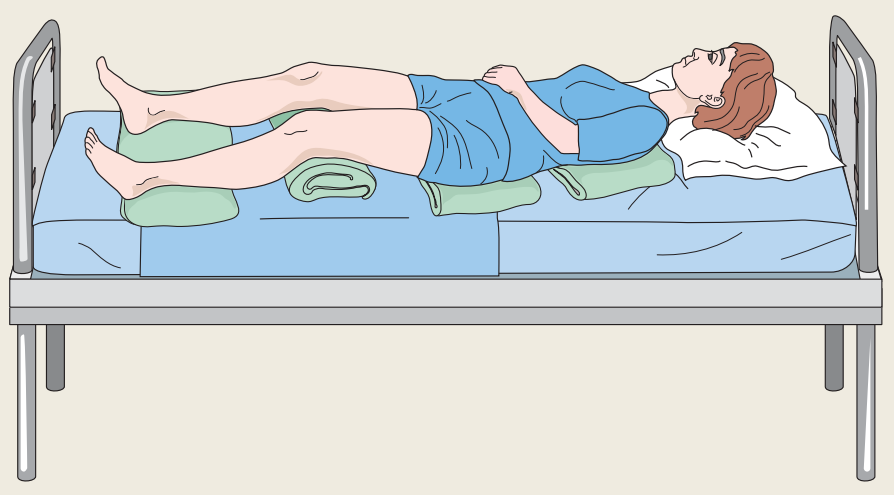



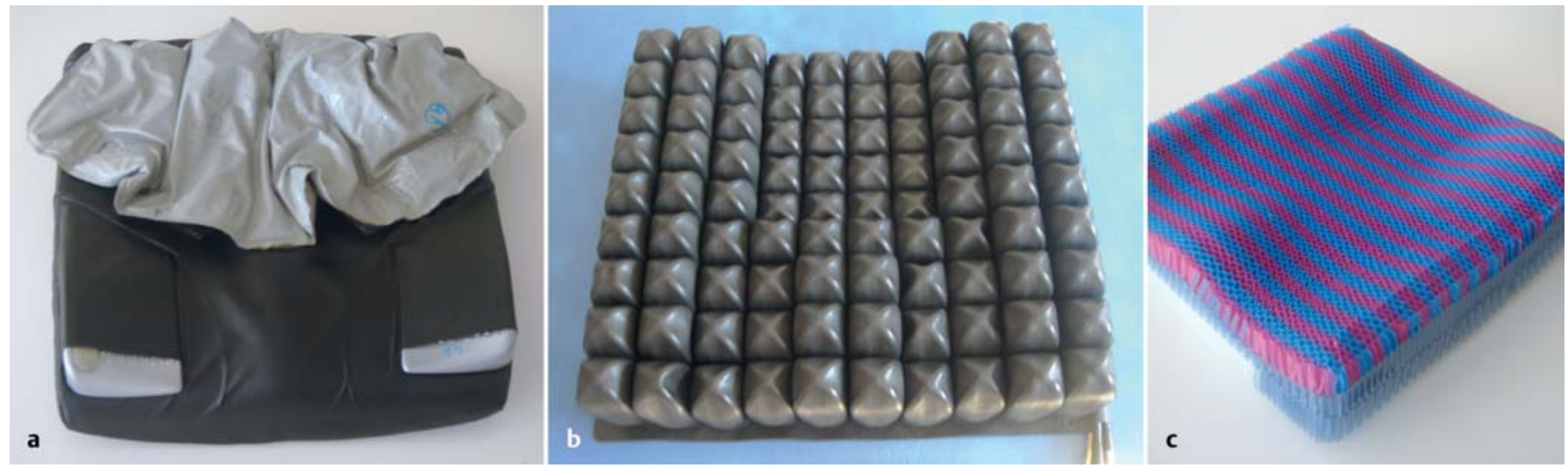

Abb. 6 - Unterschiedliche druckentlastende Spezialsitzkissen. a Gelkissen. b Luftkissen. c Wabenkissen.

Die „magische Zwei-Stunden-Regel“ ist allerdings wissenschaftlich nicht unterstützt. Zur weiteren Risikoeinschätzung sollte mit einer Belastungsprobe von 2 Stunden begonnen werden. Treten nach 2 Stunden Hautrötungen auf, muss das Zeitintervall verkürzt werden. Dies ist auch von der genutzten Unterlage abhängig. Somit kann nach mehreren Tests die individuelle Zeitspanne der Lagerung angepasst werden. Es sollten auch die Motivation und die physischen Möglichkeiten, dass sich ein Patient selbst mobilisiert, eruiert werden und in einen Lagerungsplan eingearbeitet werden.

Hierbei ist eine vollständige Druckentlastung des betroffenen Areals durch dementsprechende Lagerung und Hilfsmittel durchzuführen. Durch Mobilisation im Bett, Kinästhetik und Nutzen von körpereigenen Ressourcen kann das Risiko minimiert werden. Ergänzend werden Lagerungskissen, spezielle weiche Matratzen aus Schaumstoff oder Mikroglaskugelsysteme (Sandbett) eingesetzt. Luftmatratzen mit Wechseldrucksystemen, die eine zyklisch alternierende Druckentlastung gewährleisten, werden bei Hochrisikopatienten oder nach plastischer Deckung eingesetzt, um Druck- und Scherkräfte zu minimieren. Ein Umlagern ist bei diesen Systemen nicht mehr nötig, da der Auflagedruck unter $20 \mathrm{mmHg}$ liegt.

Der unmittelbare Kontakt von Knochenprominenzen mit der Unterlage sollte vermieden werden. Um das zu erreichen, gibt es verschiedene Lagerungstechniken (Abb. 5, S. 13).

Wichtigstes Gebot hierbei: Ein Patient sollte niemals auf seinem Dekubitus gelagert werden.
Rollstuhlversorgte Patienten mit einem Dekubitus am Gesäß müssen mit druckentlastenden Spezialkissen (Abb.6) versorgt werden und dazu angeleitet werden, ihr Gesäß alle 15 Minuten zu entlasten.

Die Bekämpfung von Risikofaktoren und spezielle Lagerungen allein heilen aber keinen Dekubitus. Zum Bereich der konservativen Therapie eines Dekubitus zählt deshalb auch die Wundbehandlung an sich. Nach T.D. Turner 1979 muss ein Wundverband 7 Kriterien erfüllen:

- Entfernung von überschüssigem Exsudat und toxischen Bestandteilen,

- Aufrechterhaltung eines feuchten Milieus im Wundbereich,

- Gewährleistung des Gasaustauschs,

- thermische Isolierung der Wunde,

- Schutz vor Sekundärinfektion durch Undurchlässigkeit für Mikroorganismen von außen,

- Ermöglichung eines atraumatischen Verbandwechsels,

- keine Abgabe von Fasern oder anderen Fremdstoffen.

\section{- Wiederherstellung eines normalen Wundmilieus}

Um die Regeneration eines gestörten Gewebes zu beschleunigen, braucht es ein gesundes Wundklima inklusive Feuchtigkeit, Körperwärme, Sauerstoff.

Abhängig von der Wundheilungsphasen muss mit verschiedenen Wundauflagen gearbeitet werden (Abb. 7).

Die feuchte Wundbehandlung fördert die Migration von Epithelzellen zum Wundgrund. Durch Okklusion einer Wunde wird ein feuchtes Milieu erreicht, und durch Sinken des Gewebesauerstoffs wird die Angiogenese angeregt. Auch wird der Abbau von Wundbelägen durch 


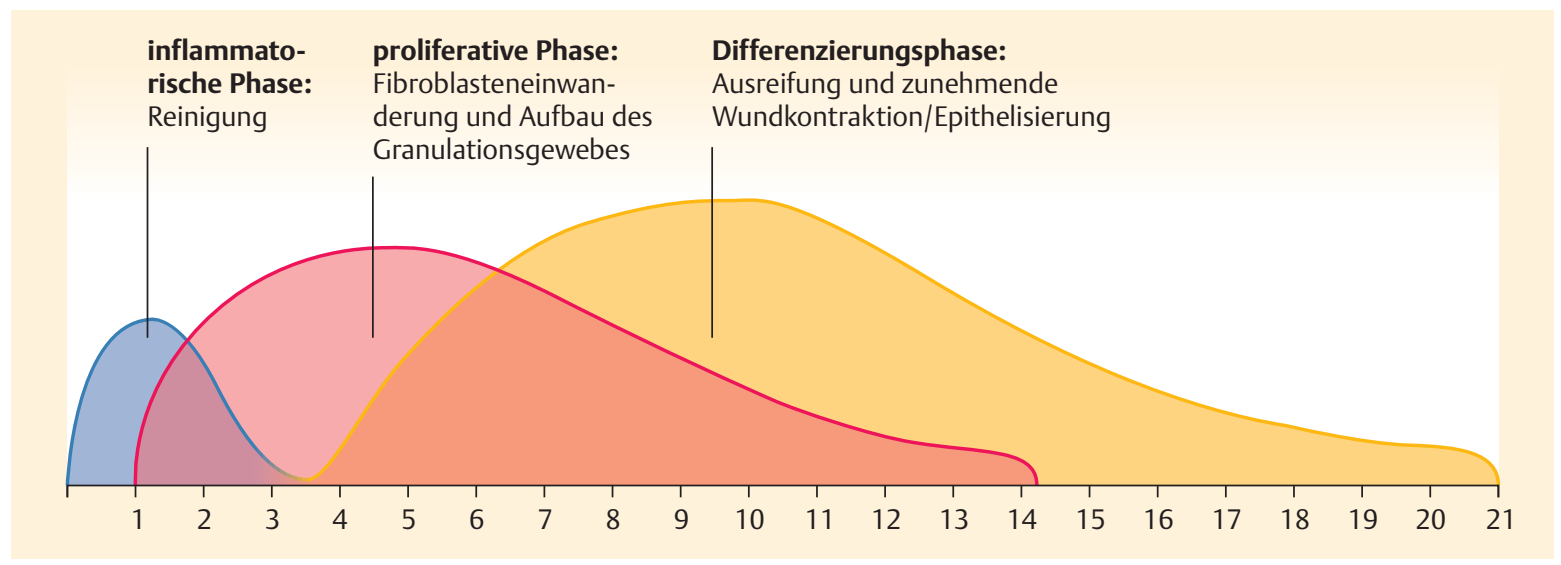

Abb. 7 - Zeitablauf der Wundheilungsphasen.

Leukozyten, Makrophagen und körpereigene Enzyme beschleunigt.

Kontraindikationen für okklusive Verbände sind Fisteln, Infektionen, freiliegende Knochen- und Sehnenstrukturen. Auch ischämische Wunden bei verminderter Perfusion sind für feuchte Wundtherapie nicht geeignet. Beim Verbandswechsel sollten keine desinfizierende Mittel eingesetzt werden, da diese zytotoxisch wirken.

Die Entfernung von überschüssigem Exsudat und toxischen Bestandteilen sowie von Hypergranulationen, Fibrinbelägen oder Nekrosen wird unter dem Begriff Débridement zusammengefasst. Das Abtragen von Nekrosen und Fibrinbelägen ist wesentlich, um den Wundgrund und die Wundtiefe beurteilen zu können. Zusätzlich verzögern diese Beläge die Wundheilung und fördern bakterielles Wachstum.

Es gibt verschiedene Typen des Débridements:

- mechanisches Débridement,

- chirurgisches Débridement,

- enzymatisches Débridement,

- biochirurgisches Débridement,

- autolytisches Débridement.

Unter mechanischem Débridement versteht man das Auflegen und leichte Andrücken von sterilen ggf. mit Wundspüllösung oder Antiseptika getränkten Kompressen. Der Belag bleibt an der Kompresse haften. Unter Verwendung jeweils neuer Kompressen wird dieses Vorgehen so oft wiederholt, bis keine Rückstände mehr anhaften.

Die schnellste und effektivste Wundreinigung ist das chirurgische Débridement. Hierbei besteht die Möglich- keit, auch tiefe Wunden in kürzester Zeit vollständig zu reinigen. Wichtig ist, dass mit scharfen Instrumenten gearbeitet wird, um Quetschungen und Schürfungen durch unscharfe Instrumente und infolgedessen ein Gewebetrauma zu verhindern.

Beim enzymatischen Débridement nutzt man das Bestreben der Enzyme, nekrotisches Gewebe abzubauen. Dabei werden in Interaktion mit Proteinen Gewebetrümmer und weiches nekrotisches Material verflüssigt und dann von einer Wundauflage aufgenommen. Enzyme wirken nur bei weichen Wundauflagen und bedürfen häufiger Verbandswechsel, was einen hohen pflegerischen Aufwand impliziert. Da es effektivere und kostengünstigere Alternativen gibt, ist die Wundreinigung mit Enzymen größtenteils verlassen worden.

Die steril gezüchtete Made Lucilia sericata findet beim biochirurgischen Débridement ihre Anwendung. Sie beeinflusst die Wunde in dreierlei Hinsicht. Es kommt:

- zu einer Wundreinigung,

- zur Keimvernichtung,

- zur Stimulation der Abheilung.

Das Funktionsprinzip der Madentherapie liegt darin, dass die Maden ein extrakorporales Verdauungssystem besitzen. Die Maden, zumeist in Biobags appliziert, sondern ihren Speichel auf die Wunde ab. Der Speichel löst in der Wunde avitales Gewebe auf. Der entstandene Verdauungsbrei aus avitalem Gewebe und Madenspeichel wird dann von der Made wieder aufgenommen. Von großem Vorteil ist, dass durch den Speichel kein vitales Gewebe angegriffen wird. Es kann durch den Speichel der Wunde zu Mikroblutungen kommen, sodass auf eine Madentherapie in der Nähe von großen Gefäßen verzichtet werden sollte. Da die Maden nach einer gewissen Zeit gesättigt 
sind, sollten sie maximal 3-5 Tage auf der Wunde belassen werden. Als Dosierung werden 5-10 Maden $/ \mathrm{cm}^{2}$ empfohlen. Zusätzlich verwendete Lokaltherapeutika werden von den Larven oftmals nicht überlebt, sodass eine Wunde vor einer geplanten Madentherapie von allen lokalen Medikamenten befreit und gereinigt werden muss.

Die Anwendung von Hydrogelen in Gelform bewirkt ein autolytisches Débridement. Es kommt zu einem Aufweichen von Nekrosen und Belägen, indem durch Rehydratation ihre innere Festigkeit zerstört wird. Um die sich lösenden Beläge aufzufangen, bedarf es eines Sekundärverbands.

Nach dem Débridement entscheidet der aktuelle Zustand des Dekubitus über die weitere Wundversorgung. Unterschieden wird hier nach den unterschiedlichen Wundheilungsphasen:

- der tiefe und/oder unterminierte Dekubitus,

- der granulierende Dekubitus,

- der epithelisierende Dekubitus.

Beim tiefen Dekubitus ist es unerlässlich, dass das Verbandsmaterial Kontakt zum Wundgrund hat, um zu verhindern, dass sich die Wunde über einem nicht zu beobachtenden Hohlraum verschließt und sich eine Infektion unbemerkt entwickeln kann. Zum Auskleiden der Wundhöhle eignen sich Kalziumalginate, Hydrofaser, feinporige Polyurethanschaum-/Hydropolymerverbände und sog. Cavity-Produkte. Nachdem die Höhle austamponiert wurde, bedarf der Dekubitus einer Abdeckung im Sinne eines semipermeablen Transparentfolien-, Hydrokolloid-, Hydropolymer- oder Hydrokapillarverbands.

- Alginate werden aus gezüchteten Braunalgen hergestellt. In ihnen sind Alginsäure, Kalzium und Spurenelemente enthalten. Wenn Alginate mit dem Wundsekret in Kontakt kommen, erfolgt ein Ionenaustausch. Hierbei gibt das Alginat Kalziumionen ab und nimmt Natriumionen aus dem Exsudat auf. Darunter kommt es zu einer Strukturveränderung des Alginats. Vom festen Kalziumalginat wird es zum löslichen, gelförmigen Natriumalginat. Das abgegebene Kalzium wirkt in der Wunde blutstillend. Natriumalginat ist als Gel sehr aufnahmefähig und nimmt neben Exsudat auch Zelltrümmer auf. Zu beachten ist, dass auf Druck die absorbierte Flüssigkeit wieder abgegeben wird. Dies ist beim Einbringen der Alginate in die Wunde auch im Hinblick auf die Entstehung von Wundrandmazerationen zu bedenken. Da Alginate körperfremde Stoffe sind, muss beim Verbandswechsel auf die vollständige Entfernung der Alginate geachtet werden.
- Als Alternative zu Alginaten können Wundhöhlen mit Hydrofaser tamponiert werden. Diese bestehen aus Natriumcarboxymethylzellulose und verwandeln sich nach Kontakt mit Wundexsudat in ein transparentes Gel. Beim Aufquellen dehnt sich das Gel lediglich in vertikaler Richtung aus, sodass das Risiko einer Wundrandmazeration gemindert ist. Da Hydrofasern das bis zu 25-Fache ihres Eigengewichts an Exsudat aufnehmen können, muss auf die Gefahr der Wundaustrocknung geachtet werden. Wie auch Alginate sind Hydrofasern körperfremde Stoffe und müssen beim Verbandswechsel vollständig entfernt werden.

- Als dritte Möglichkeit der Wundversorgung eines stark exsudierenden, tiefen Dekubitus sind feinporige Polyurethanschaum-/Hydropolymerverbände zu nennen. Sie bestehen aus einem feinporigen Polyurethanweichschaum, der das bis zu 30-Fache seines Eigengewichts an Exsudat aufnehmen kann. Zusätzlich werden Bakterien und Zelltrümmer aufgenommen. Durch die luftdurchlässige Membran ist ein Gasaustausch ermöglicht. Die thermoisolierende Funktion sichert ein gleichbleibend feuchtes und warmes Wundklima. Nach Aufnahme von Exsudat quellen die Verbände unterschiedlich stark auf, sodass kleine Wundunebenheiten ausgeglichen werden können. Bei Sättigung kann es zu Mazerationen kommen.

Der granulierende Dekubitus zeichnet sich durch eine saubere, gekörnte tiefrote Wunde mit guter Durchblutung, ein feucht glänzendes Erscheinungsbild und eine abnehmende Exsudation aus. Wesentliches Ziel der Wundversorgung ist in dieser Phase der Schutz des frischen Gewebes und der Schutz vor Sekundärinfektionen. Hierfür gibt es mehrere Möglichkeiten der Wundabdeckung: Hydrokolloidverbände, feinporiger Polyurethanschaum-/Hydropolymerverband, Hydrokapillarverband, Hydrogelkompressen und transparente Hydroaktivverbände.

- Bei Hydrokolloidverbänden handelt es sich um semiokklusive Wundauflagen, die auf mäßig bis schwach exsudierenden Wunden zum Einsatz kommen. Ihre Aufgabe besteht weniger darin, Exsudat aufzunehmen, als vielmehr die Granulation zu fördern, die Wunde feucht zu halten, Zelltrümmer aufzunehmen und vor einer Sekundärinfektion zu schützen. Die Hydrokolloide bestehen aus einer hydrophoben Polymermatrix, in die hydrophile Teilchen eingebettet sind. Nach Kontakt mit Exsudat bildet sich ein gelbliches Gel, das unter der Wundauflage als Blase imponiert. Wenn die Blase den Umfang der Wunde überschreitet, ist der Verband zu wechseln und eine Wundspülung mit Ringer-Lösung oder $\mathrm{NaCl}$ 0,9\% durchzuführen. 
- Der feinporige Polyurethanschaum-/Hydropolymerverband wurde bereits im Abschnitt der Wundbehandlung eines tiefen Dekubitus besprochen.

- Als Alternative zu Hydrokolloidverbänden sind die aus hydrokapillaren Wundkissen bestehenden Hydrokapillarverbände zu nennen. Diese Verbände absorbieren Zelltrümmer, Bakterien und überschüssiges Wundexsudat. Diese Verbände können große Mengen an Exsudat aufnehmen und sind deshalb sowohl für stark exsudierende Wunden als auch für eine längere Auflagedauer geeignet. Bei weniger stark exsudierenden Wunden muss auf ein Austrocknen geachtet werden.

- Bei geringer Exsudation einer granulierenden Wunde eignen sich Hydrogelkompressen. Diese Kompressen bestehen zu 15-95\% aus Wasser und dienen als semiokklusive Wundverbände zur feuchten Wundbehandlung. Durch einen kühlenden Effekt wirken sie zugleich schmerzlindernd. Von Vorteil ist ihre Transparenz, die eine gute Wundkontrolle ermöglicht.

- Als Alternative zu Hydrogelkompressen sind transparente Hydroaktivverbände zu sehen. Hierbei wird ein anpassungsfähiges Acrylkissen von 2 semipermeablen Transparentklebefolien umschlossen. So kann das Kissen Wundexsudat aufnehmen und es zugleich per Verdunstung nach außen wieder abgeben. Diese Verbände sind nur für schwach bis mäßig exsudierende Wunden geeignet. Auch hier ist aufgrund der Transparenz eine gute Wundkontrolle möglich.

Der epithelisierende Dekubitus zeichnet sich dadurch aus, dass sich das Gewebe rosa, hellrosa bis weißlich darstellt. In dieser Phase ist es wichtig, die Oberfläche feucht zu halten, um die Wanderung der Epithelzellen zu gewährleisten. Die Wundauflage darf keine Saugwirkung entfalten, um das Austrocknen der Wunde zu vermeiden. Hauptaspekt ist das Schaffen einer langen Wundruhe und ein schonender atraumatischer Verbandswechsel. Hier sind verschiedene Verbände anwendbar: transparenter oder dünner Hydrokolloidverband, Hydrogelkompressen, transparenter Hydroaktivverband, dünner feinporiger Polyurethanschaum-/Hydropolymerverband oder semipermeable Transparentfolien.

Zu großen Teilen wurden diese Verbandsmaterialien bereits bei der Versorgung der granulierenden Wunde beschrieben.

- Die semipermeable Transparentfolie ermöglicht keinerlei Aufnahme von Exsudat. Jedoch wirkt ihre semipermeable Membran als Keimbarriere und ermöglicht durch ihre Transparenz eine gute Wundbeobachtung. Ein wesentlicher Vorteil dieser Folien für die Patienten liegt darin, dass bei ihrer Verwendung Duschen ggf. Baden ermöglicht wird.

\section{Der kontaminierte Dekubitus}

Prinzipiell kann von einer Kontamination eines Dekubitus ausgegangen werden. Eine Infektion entsteht, indem sich die Keime vermehren und durch ihre Toxine Gewebe schädigen. Dies äußert sich durch Calor, Rubor, Dolor, Eiterbildung und Geruch, systemisch mit Fieber, Schüttelfrost, Leukozytose. Je ausgeprägter ein Dekubitus kontaminiert ist, umso größer wird die Gefahr der Infektion.

Gegenmaßnahmen sind:

- ausreichende Blut- und Sauerstoffversorgung damit Leukozyten und Makrophagen, die die Phagozytose suffizient durchführen können,

- Entfernung von Nekrosen, um Nährboden zu entziehen,

- Absaugung keimbelasteten Exsudats durch Wundverbände zur Keimeliminierung.

Bei ausgeprägten lokalen Entzündungszeichen muss eine weitergehende Therapie erfolgen:

- bakteriologischer Abstrich,

- systemisches Breitbandantibiotikum (z.B. Amoxicillin/ Clavulansäure, Levofloxacin, resp. Ciprofloxacin bei Pseudomonas),

- Ruhigstellung,

- Débridement, mechanische Reinigung,

- mindestens täglicher Verbandswechsel,

- evtl. Anpassung der Antibiotikatherapie nach Antibiogramm des Abstrichs,

- keine lokalen Antibiotika, Silberpräparate sind aber erlaubt,

- keine Okklusivtherapie.

In der Granulationsphase sollte die feuchte Wundbehandlung fortgesetzt werden. Eine Wunde darf nie austrocknen, da sonst die für den Gefäß- und Gewebeaufbau notwendigen Zellen absterben. In der Epithelisierungsphase ist die feuchte Wundbehandlung fortzuführen, bei großflächigen Ulzera ist ggf. eine Unterstützung der Epithelisierung notwendig, z.B. mit Reverdin-Plastik. 


\section{Grundlagen}

\section{Anhang}

Tabelle A1

Norton-Skala (nach Seiler 2002).

\begin{tabular}{|c|c|c|c|c|}
\hline körperlicher Zustand & Inkontinenz & Aktivität & Beweglichkeit & geistiger Zustand \\
\hline 4 gut & 4 keine & 4 geht ohne Hilfe & 4 voll & 4 klar \\
\hline 3 leidlich & 3 manchmal & 3 geht mit Hilfe & 3 kaum eingeschränkt & 3 apathisch/teilnahmslos \\
\hline 2 schlecht & 2 meistens Urin & 2 rollstuhlbedürftig & 2 sehr eingeschränkt & 2 verwirrt \\
\hline 1 sehr schlecht & 1 Urin und Stuhl & 1 bettlägerig & 1 voll eingeschränkt & 1 stuporös \\
\hline Bereitschaft zur Kooperation & Alter & Hautzustand & Zusatzerkrankungen & \\
\hline 4 voll & $4<10$ & 4 normal & 4 keine & \\
\hline 3 wenig & $3<30$ & 3 schuppig & \multicolumn{2}{|c|}{3 Abwehrschwäche, Fieber, Diabetes, Anämie } \\
\hline 2 teilweise & $2<60$ & 2 feucht & \multicolumn{2}{|c|}{2 MS, Karzinom, erhöhter Hämatokrit, Adipositas } \\
\hline 1 keine & $1>60$ & 1 Allergie, Wunden, Risse & \multicolumn{2}{|c|}{1 arterielle Verschlusskrankheit } \\
\hline
\end{tabular}

Der Patient wird nach den in der Tabelle aufgeführten Kriterien bewertet, und die Punkte werden addiert. Es besteht bei 18-15 Punkten ein geringes, bei 14-13
Punkten ein mittleres, bei 12-10 Punkte ein hohes und bei $<9$ ein sehr hohes Risiko, einen Dekubitus zu erleiden.

Tabelle A2

Braden-Skala (nach Seiler 2002).

\begin{tabular}{|c|c|c|c|c|c|}
\hline & 1 Punkt & 2 Punkte & 3 Punkte & 4 Punkte & Punkte \\
\hline $\begin{array}{l}\text { sensorisches } \\
\text { Wahrnehmungs- } \\
\text { vermögen } \\
\text { Fähigkeit, lagebedingte } \\
\text { wie künstliche Reize } \\
\text { wahrzunehmen und } \\
\text { adäquat zu reagieren }\end{array}$ & $\begin{array}{l}\text { vollständig ausgefallen } \\
\text { - Keine Reaktion auf } \\
\text { Schmerzreize (auch kein } \\
\text { Stöhnen, Zucken, Greifen) } \\
\text { aufgrund verminderter } \\
\text { (nervaler) Wahrnehmungs- } \\
\text { fähigkeit bis hin zur } \\
\text { Bewusstlosigkeit oder } \\
\text { Sedierung } \\
\text { oder } \\
\text { Missempfindungen/ } \\
\text { Schmerzen werden über } \\
\text { den größten Körperanteil } \\
\text { nicht wahrgenommen. }\end{array}$ & $\begin{array}{l}\text { stark eingeschränkt } \\
\text { - Reaktion nur auf starke } \\
\text { Schmerzreize, Missemp- } \\
\text { findungen können nur } \\
\text { über Stöhnen oder } \\
\text { Unruhe mitgeteilt werden } \\
\text { oder } \\
\text { - sensorisches Empfinden } \\
\text { stark herabgesetzt. } \\
\text { Missempfindungen/ } \\
\text { Schmerzen werden über } \\
\text { die Hälfte des Körpers } \\
\text { nicht wahrgenommen. }\end{array}$ & $\begin{array}{l}\text { geringfügig eingeschränkt } \\
\text { - Reaktion auf Ansprechen; } \\
\text { Missempfindungen bzw. } \\
\text { das Bedürfnis nach Lage- } \\
\text { rungswechsel können nicht } \\
\text { immer vermittelt werden } \\
\text { oder } \\
\text { - sensorisches Empfinden } \\
\text { teilweise herabgesetzt. } \\
\text { Missempfindungen/ } \\
\text { Schmerzen werden in } \\
1 \text { oder } 2 \text { Extremitäten } \\
\text { nicht wahrgenommen. }\end{array}$ & $\begin{array}{l}\text { nicht eingeschränkt } \\
\text { - Reaktion auf Ansprechen; } \\
\text { Missempfindungen/ } \\
\text { Schmerzen werden wahr- } \\
\text { genommen und können } \\
\text { benannt werden. }\end{array}$ & \\
\hline
\end{tabular}


Braden-Skala. (Fortsetzung)

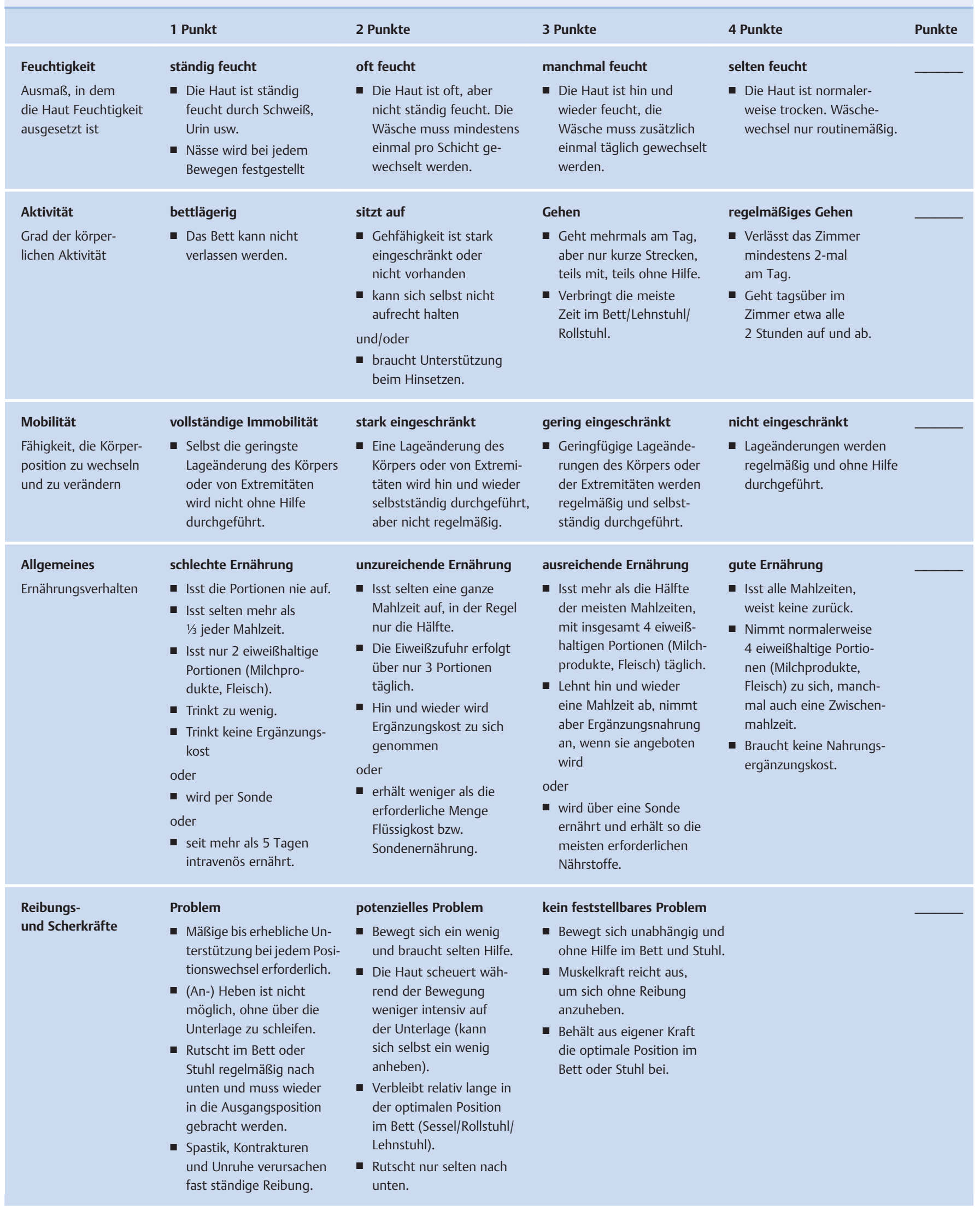




\section{Grundlagen}

Der Patient wird zu jedem Item mit einem Punktwert beurteilt. Die einzelnen Punktwerte werden addiert. Bei einer Summe von 18 oder weniger Punkten besteht ein erhöhtes Dekubitusrisiko. Je geringer die Punktzahl desto höher das Dekubitusrisiko.

\section{Tabelle A3}

Chirurgische Dekubitusklassifikation (mod. nach Daniel 1979).

\begin{tabular}{|l|l|}
\hline Stadium 0 & wegdrückbare Hautrötung \\
\hline Stadium I & $\begin{array}{l}\text { scharf begrenzte Hautrötung bei intakter Haut, die sich nicht „wegdrücken“ lässt. Auch kann sich eine Überwärmung, } \\
\text { eine Verhärtung oder ein Ödem darstellen. Bei Personen mit dunkler Hautfarbe kann sich eine Entfärbung der Haut } \\
\text { zeigen. Bei konsequenter Druckentlastung verblasst die Rötung nach einigen Stunden bis Tagen. }\end{array}$ \\
\hline Stadium II & $\begin{array}{l}\text { Teilverlust der Epidermis bis hin zur Dermis } \\
\text { oberflächliches Ulkus, das sich klinisch als Abrasion, Blase oder flacher Krater zeigt }\end{array}$ \\
\hline Stadium III & Ausdehnung bis in das subkutane Fettgewebe \\
\hline Stadium IV & Schädigung aller Hautschichten (Epidermis, Dermis und Subkutis) mit Muskelbeteiligung ohne Knochen \\
\hline Stadium V & $\begin{array}{l}\text { kompletter Verlust der gesamten Haut über alle Schichten mit ausgedehnten Gewebenekrosen und Schädigung } \\
\text { zer Muskeln, Sehnen und Knochen }\end{array}$ \\
\hline
\end{tabular}

Tabelle A4

Stadieneinteilung des Wundzustands (nach Seiler 1979).

Stadium A Wunde „sauber“, Granulationsgewebe, keine Nekrosen

Stadium B Wunde schmierig belegt, Restnekrosen, keine Infiltration des umgebenden Gewebes

Stadium C Wunde wie Stadium B mit Infiltration des umgebenden Gewebes 


\section{Therapie und Prophylaxe von Dekubitalulzera - Teil 1}

Tabelle A5

Gegenüberstellung der gebräuchlichen Klassifikationen.

\begin{tabular}{|c|c|c|c|c|c|c|}
\hline & & Daniel (1979) & & $\begin{array}{l}\text { NPUAP (National Pressure } \\
\text { Ulcer Advisory Panel 1989) }\end{array}$ & & Seiler (1979) \\
\hline & & $\begin{array}{l}\text { orientiert sich an } \\
\text { Hautschichten } \\
\text { chirurgische Klassifikation } \\
\text { gilt für jede Wunde }\end{array}$ & & hat Seiler 1989 abgelöst & & in Fachliteratur überholt \\
\hline 1. Grad & Oberhaut & $\begin{array}{l}\text { Erythem } \\
\text { scharf begrenzt, schmerzlos, } \\
\text { reversibel, umschriebene } \\
\text { Hautrötung bei intakter } \\
\text { Epidermis }\end{array}$ & Oberhaut & $\begin{array}{l}\text { scharf begrenzte Hautrötung } \\
\text { bei intakter Oberhaut. Ödem- } \\
\text { bildung, Verhärtung, lokale } \\
\text { Überwärmung }\end{array}$ & Oberhaut & $\begin{array}{l}\text { umschriebene Hautrötung, } \\
\text { absolut intakt } \\
\text { lässt sich durch Fingerdruck } \\
\text { nicht wegdrücken }\end{array}$ \\
\hline 2. Grad & Lederhaut & $\begin{array}{l}\text { Blasenbildung der Kutis, } \\
\text { oberflächlich Ulzeration } \\
\text { der Epidermis bis Dermis }\end{array}$ & Lederhaut & $\begin{array}{l}\text { Teilverlust der Haut } \\
\text { Epidermis bis Dermis } \\
\text { sind geschädigt } \\
\text { klinisch: Blase }\end{array}$ & Lederhaut & $\begin{array}{l}\text { Blase, Hautabschürfung oder } \\
\text { flaches Geschwür. Bei Auf- } \\
\text { platzen der Blase entsteht eine } \\
\text { nässende, infektionsanfällige } \\
\text { Schädigung der Epidermis bis } \\
\text { Dermis }\end{array}$ \\
\hline 3. Grad & Unterhaut & Ulkus bis Subkutis & Unterhaut & $\begin{array}{l}\text { Verlust aller Hautschichten } \\
\text { oder Nekrosen von sub- } \\
\text { kutanem Gewebe, bis zu } \\
\text { Faszien reichend } \\
\text { Faszie und Muskel nicht } \\
\text { betroffen }\end{array}$ & $\begin{array}{l}\text { Unterhaut, } \\
\text { Muskel }\end{array}$ & $\begin{array}{l}\text { tiefes offenes Geschwür, } \\
\text { Muskel, Sehnen betroffen }\end{array}$ \\
\hline 4. Grad & Muskel & $\begin{array}{l}\text { Ulzeration bis Faszie } \\
\text { z.T. mit Muskelbefall }\end{array}$ & $\begin{array}{l}\text { Muskel, } \\
\text { Knochen }\end{array}$ & $\begin{array}{l}\text { Verlust aller Hautschichten } \\
\text { mit ausgedehnter Nekrose } \\
\text { mit Beteiligung von Muskeln } \\
\text { und Knochen, Sehnen, } \\
\text { Gelenkkapseln }\end{array}$ & Knochen & $\begin{array}{l}\text { tiefes offenes Geschwür mit } \\
\text { Schädigung aller Gewebe- } \\
\text { schichten mit Knochen- } \\
\text { beteiligung }\end{array}$ \\
\hline 5. Grad & Knochen & $\begin{array}{l}\text { Befall von Knochen, } \\
\text { Gelenken, Beckenorgane } \\
\text { (Rektum, Vagina usw.) }\end{array}$ & & & & \\
\hline
\end{tabular}




\section{Quellenangaben}

Anders J, Heinemann A, Leffmann C et al. Dekubitalgeschwüre Pathologie und Primärpravention. Dtsch Ärztebl 2010; 107: 371 - 382

Bennett L, Kavner D, Lee BK et al. Skin stress and blood flow in sitting paraplegic patients. Arch Phys Med Rehabil 1984; 65: 186-190

Daniel RK, Hall EJ, MacLead MK. Pressure sorces - a reappraisal. Ann Plast Surg 1979; 3: 53-63

Die phasengerechte Wundbehandlung des Dekubitalulkus. Hartmann medicaledition 2005; 3: 2.3 - 78.79

von Goossens RHM, Zegers R, Hoek van Dijke GA et al. Influence of shear on skin oxygen tension. Clin Physiol 1994; 14: 111-118

Grundlagen zur Erarbeitung von Leitlinien zur Dekubitusbehandlung. Hartmann WundForum 2001; 3: 27-33

Holle G, Peek A. Stellenwert der VAC-Therapie beim akuten und chronischn Weichteiltrauma. Hefte zu „Medizinisch Orthopädische Technik“ 2008; 6: 49-56

Seiler O. Dekubitus - Pathogenese und Prophylaxe. Hartmann WundForum 2002; 3: 9-15

Shea J. Pressure sores. Classification and management. Clin Orthop 1975; 112: $89-100$

Turner TD. Hospital usage of absorbent dressings. Pharm J 1979; 222: $421-426$

Young T. Pressure sores: incidence, risk assessment and prevention. Br J Nurs 1997; 6: 319-322

Zäch GA, Koch HG. Paraplegie, Ganzheitliche Rehabilitation. Basel: Karger; 2005: 209-221

\section{Korrespondenzadresse}

Dr. med. M. Schempf

Nova Clinic Biberach an der Riß

Eichendorffweg 5

88400 Biberach

Telefon: 0049-7351-44490

Fax: $\quad$ 0049-7351-444911

E-Mail: schempf@nova-clinic.de 


\section{CME-Fragen}

In welchem Stadium nach

Daniel entstehen Blasen?

\section{CME•thieme.de}

\section{CME-Teilnahme}

- Viel Erfolg bei lhrer CME-Teilnahme unter http://cme.thieme.de

- Diese Fortbildungseinheit ist 12 Monate online für eine CME-Teilnahme verfügbar.

- Sollten Sie Fragen zur Online-Teilnahme haben, unter http://cme.thieme.de/hilfe finden Sie eine ausführliche Anleitung.

Wie hoch muss der Gegendruck einer Auflagefläche mindestens sein, damit ein Dekubitus

A $<20 \mathrm{mmHg}$.
B $25-35 \mathrm{mmHg}$. entstehen kann?
A Stadium I.
B Stadium II.
C Stadium III.
D Stadium IV.
E Stadium V.

Für welche anatomische Region eignet sich v.a. die $135^{\circ}$-Lagerung?
A Morbus Parkinson.

B Kachexie.

C Infektion.

D Steroidtherapie.

E Diabetes.
Was ist die Grundtherapie zur Prophylaxe eines Dekubitus?
A Druckmassage.
B Druckverringerung.
C Längere Zeiten der Lagerung.
D Kälteapplikation.
E Sterile Abdeckung des Ulkus.
A Ellenbogen.
B Frontaler Kopf.
C Os sacrum.
D Kniescheibe.
E Spina iliaca anterior superior. 


\section{Grundlagen}

Welcher Risikofaktor zählt

nicht zu den Anforderungen

der NICE-Empfehlung?
A Bewusstseinsgrad.
B Krankheitsschwere.
C Alter.
D Hygiene.
E Hautzustand.

Was ist ein wichtiges Therapieziel in der Dekubitustherapie?
A Verbesserung des Allgemeinzustands, z. B. Reduktion der Malnutrition.

B Verkürzung der Liegeverweildauer durch kurzzeitigen Wundverschluss.

C Verminderung der Schmerzwahrnehmung durch geeignete Schmerzmedikamente.

D Photodokumentation.

E Anpassung der Liegeverweildauer auf einem Dekubitus abhängig von dessen Größe und Qualität.

\begin{tabular}{l|l} 
Ab welchem Schweregrad & A Grad 1 \\
eines Dekubitus wird mit & B Grad 2 \\
einer plastischen Weichteil- & C Grad 3 \\
deckung begonnen? & D Grad 4 \\
& E Grad 5
\end{tabular}

Welches ist kein Kriterium zur konservativen Wundbehandlung nach T.D. Turner?
A Entfernung von überschüssigem Exsudat und toxischen Bestandteilen.

B Wundbehandlung mit Zucker zur Reduktion von nässenden Wunden.

C Ermöglichung eines atraumatischen Verbandswechsels.

D Verbesserung des Gasaustauschs.

E Keine Abgabe von Fasern oder anderen Fremdstoffen.
Welche Aussage trifft zu?
B Hydrofasern können in Wunden belassen werden, da sie körperähnliche Stoffe enthalten.

C Das abgegebene Kalzium bei Alginaten hat den Nachteil der erhöhten Blutungsgefahr in Wunden.

D Hydrogelkompressen eignen sich für stark sezernierende Wunden.

E Feinporige Polyurethanschaum-/Hydropolymerverbände haben eine thermoisolierende Funktion. 